\title{
Raus aus der engen Schublade beim Kunden!
}

In der falschen Schublade versorgt zu sein, ist meistens das Problem des Anbieters und weniger des Kunden. Der Kunde bezieht nur wenige Leistungen, auch wenn er grundsätzlich weitere Produkte und Services von diesem Lieferanten bestellen könnte. Dieser Beitrag orientiert sich am B2B-Marketing und -Vertrieb und thematisiert ein unterschätztes Problem.

Christian Belz 
Der Kunde hat so viele Möglichkeiten, um seine Produkte und Dienstleistungen zu beschaffen, dass er die Leistungsfähigkeit der Lieferanten weder erkunden noch berücksichtigen muss. Die Sortimente der Lieferanten überschneiden sich stark. Recht willkürlich bedient sich der Kunde beim einen Lieferanten mit einigen Produkten und beim anderen mit weiteren Leistungen. Obschon er eigentlich alles bei einem beziehen könnte. Manchmal wechselt er ohne wirklichen Anlass oder ein zufälliger Bedarf führt zu Zusatzbestellungen.

Es gibt eher rationale Gründe des Kunden, den Lieferanten in einer kleinen Schublade zu belassen. Gewohnheiten erleichtern sein Leben und vermindern neue Recherchen, interne Überzeugung, Verhandlungen und Abläufe. Zudem werden mehr drängende Lieferanten berücksichtigt. Das Risiko ist gestreut. Zwar gibt es auch die gegenläufige Tendenz, die Lieferanten zu konzentrieren und den Bedarf zu bündeln. Oft bezieht sich das aber nur auf bestimmte Warengruppen und Treiber ist die Kostensenkung.

Dazu kommen mentale Schranken. Der Lieferant steht einfach für seine bisherigen Lieferungen, dass er mehr tun könnte, steht einfach nicht im „Mindset“ des Kunden. Dabei kann er mit dem Lieferanten hoch zufrieden sein, es kommt ihm aber gar nicht in den Sinn, die Geschäftsbeziehung zu erweitern.

Bisherige Leistungen bewirken auch, ob ein Lieferant vom Kunden als schlank und günstig beurteilt wird oder für eine Know-how- und serviceintensive Zusammenarbeit taugt. Oft berücksichtigt er dabei nicht, wenn Anbieter parallel für eine schlanke bis extensive Zusammenarbeit kompetent sind. Jeder Kunde hat eine eigene explizite oder implizite Liste, für welche Leistungen er welche Lieferanten berücksichtigt.

Aus der Sicht des Kunden bedeutet das: Die Rolle des Lieferanten ist für ihn oft sehr eng interpretiert. Was er einmal bezogen hat, prägt die Geschäftsbeziehung stark.

\section{Die Sicht des Anbieters}

Besorgt analysiert der Lieferant seinen geringen „Share of wallet" beim Kunden. Anbieter sind auch oft nach Sparten aufgeteilt und stellen fest, dass nur eine Sparte mit dem Kunden zusammenarbeitet, obschon auch die übrigen Bereiche bei ihm Potenzial erkennen. Damit verlagert sich die Diskussion darauf, wie sich das Cross Selling steigern lässt: Eine Sparte soll auch für die weiteren (vor)verkaufen.

Generalisten im Verkauf für mehrere Sparten können zwar Synergien in der Kundenbearbeitung ausschöpfen, nur sind sie auch weniger kompetent und rasch auf Spartenunterstützung angewiesen oder sie blitzen dadurch beim Kunden ab. Die Spartenorganisation hat also auch Vorteile. So werden die Lieferungen getrennt verhandelt und die Preiszugeständnisse sind geringer. Es mag auch ergiebig sein, mit mehreren und kleinen Lieferungen nicht in den Fokus des strategischen Einkaufs beim Kunden zu geraten. Ein strategischer Lieferant zu sein, bringt nicht nur Vorteile. Zudem verankern Mehrspartenbeziehungen den Lieferanten besser beim Kunden und Prob-

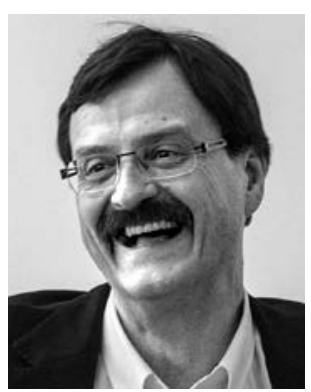

Prof. Dr. Christian Belz

ist Ordinarius für Marketing an der Universität St. Gallen und Direktor am Institut für Marketing;www.ifm.unisg.ch

Christian Belz

Universität St. Gallen, Schweiz

E-Mail:christian.belz@unisg.ch 
leme in einem Bereich übertragen sich nicht automatisch auf alle. Die Risiken und Chancen sind gestreut. Zudem ist es einfacher, Sparten zu führen und das Know-how für Kunden zu bündeln. Auch entscheiden Kunden oft sehr dezentral, so dass es für den Lieferanten viele Ansprechpersonen gibt, die ohnehin separat bearbeitet werden müssen.

Besonders bei neuen Kunden versuchen Anbieter oft, erst einmal ins Geschäft zu kommen. Sie hoffen, später von der Einzellieferung, in eine breite Zusammenarbeit vorzustossen. Beginnt jedoch der Lieferant klein, so bleibt er oft auch klein. Es ist deshalb gefährlich, nur 20 Prozent eines möglichen Geschäfts mit neuen Kunden abzuholen. Deshalb argumentiere ich auch, dass bei neuen Kunden mehr gesät werden müsste, es geht nicht nur um die Jagd und den Einstieg.

Jede Sparte nutzt die enge Zeit mit dem Kunden möglichst verkaufswirksam. Das heisst, sie konzentriert sich auf wenige und eigene Leistungen, die sie selbst beherrscht. Dort fühlt sich auch der Verkauf kompetent. Würden noch weitere Möglichkeiten in der eigenen Unternehmensgruppe eingebracht, könnte der Kunde zurück schrecken oder es wird ihm zu aufwendig und er kauft gar nichts mehr.

Die Sicht des Lieferanten ist also durchaus widersprüchlich.

\section{Die Lösungen}

Lösungen bewegen sich in den aufgezeigten Spannungen und sind deshalb oft nicht eindeutig:

Unternehmensportfolio kommunizieren: Marketing und Vertrieb müssen das Leistungsportfolio beim Kunden aktiv kommunizieren. Dazu gehören Produkte ebenso wie Services, schlanke Formen der Zusammenarbeit mit Kunden ebenso wie eine intensive Kooperation. Die Herausforderung ist dabei gross, weil Kunden naturgemäss zu engen Beurteilungen neigen. Im vielfältigen B2B-Marketing bewährt es sich beispielsweise, geschickt mit Kunden-Cases umzugehen. Auch der Internetauftritt ist dafür wichtig.

\section{Zusammenfassung}

- Oft gelingt es dem Anbieter nicht, seine Leistungsfähigkeit genügend breit für den Kunden einzubringen. Die Zusammenarbeit bleibt zu schmal.

- Verschiedene Lösungen reichen von Positionierung, Erweiterungsprozessen für bestehende Kunden, Cross Selling bis Kundenorganisation.
Kundenpotenziale abschätzen: Mit welchen Kunden gelingt es, die Geschäfte massgeblich auszuweiten? Welche Kunden entscheiden zentral? Welche Kunden halbieren ihre Lieferantenzahl? Wie wirken sich grössere Geschäfte auf die Margen aus? Flächendeckende Ansätze sind meistens unmöglich. Bei einem geringen Share of wallet und einem hohen Cross-Selling-Potenzial sind immer viele Wettbewerber im Spiel, die es vorerst auszuschalten gilt. Die Möglichkeiten realistisch abzuschätzen bleibt schwierig, oft führen entsprechende Analysen zu einer zu positiven, ja illusionären Einschätzung.

Kundenvorteile entwickeln: Der Lieferantenvorteil genügt nicht. Was sind die Vorteile des Kunden, wenn er mehr vom Lieferanten bezieht und mit mehreren seiner Sparten zusammenarbeitet? Reine Preisvorteile des Kunden möchte der Lieferant ja vermeiden. Deshalb gilt es, andere und relevante Kundenvorteile zu erfassen und Lösungen zu entwickeln.

Positionierung bei neuen Kunden: Marketing und Verkauf sollen das eigene Unternehmen bei neuen Kunden eher breiter positionieren, ohne gezielte Geschäfte zu verhindern. Es genügt nicht, den Fuß in der Türe zu haben.

Kundenbeziehung erweitern: Für aktuelle Kunden braucht es klare Prozesse, um bestehende Geschäfte zu erweitern. Dazu gilt es, erfolgreiche Erweiterungen zu analysieren und Vorgehensvarianten zu bestimmen, das Unternehmen mit der Kundenorganisation zu vernetzen und neue Projekte mit Kunden anzustossen.

Cross Selling: Manche Appelle zu mehr Cross Selling versanden rasch und bleiben unrealistisch. Es ergeben sich Querbezüge zu den vorstehenden Hinweisen: Realistisch abgeschätzte Potenziale des Cross Selling, Kundenselektion, flankierendes Marketing, verkaufswirksame Nutzung der Customer Face Time. Zudem gilt es, Verkaufsführung und Incentives anzupassen und das Zusammenspiel des Verkaufs mit internen Abteilungen und Spezialisten zu erleichtern.

Cross Selling und Key Account Management sind eine Vorstufe zur Kundenorganisation, dabei versucht das Unternehmen, die Bereiche für den Kunden zu koordinieren, weil es nicht nach dem Kundenbedarf aufgestellt ist.

Kundenorganisation: Plausibel ist es, ein Unternehmen nach Kunden aufzustellen, also sich beispielsweise für Branchen und Segmente zu spezialisieren. Zwar optimieren Unternehmen dabei immer noch nach Produkten und Services, Ländern, Kanälen und Funktionen. Die Kundenorientierung äussert sich aber darin, dass mehr Wertschöpfung im Unternehmen nach Kundengruppen spezialisiert ist. One 


\section{Handlungsempfehlungen}

- Erfassen Sie bestehendes und mögliches Geschäft bei Ihren Kunden.

- Analysieren Sie mit den Kunden, welche Ursachen für die schmale Zusammenarbeit wichtig sind.

- Thematisieren Sie die Problematik der „engen Schublade" mit Ihrem Verkaufsteam und entwickeln Sie neue Lösungen.

stop shopping für den Kunden ist das Schlagwort. Voraussetzung für den Erfolg ist dabei, dass diese Spezialisierung dort genutzt wird, wo auch der Kunde eher zentral entscheidet und Einkaufssynergien aktiv nutzt. Der steigende Preisdruck muss sich durch das Wachstum mit Kunden auffangen lassen.

Manchmal wird nur der Verkauf nach Kundengruppen organisiert. Generalisten im Verkauf orchestrieren die Leistung des Unternehmens für Kunden. Kritisch bleiben seine Kompetenz bei Kundenspezialisten und nochmals das Zusammenspiel zwischen Verkauf und übrigen Unternehmenseinheiten für Kunden. Kritisch ist auch, was der Verkauf bei Kunden einfädelt, weil inkompetente Verkäufer leicht mehr versprechen, als ein Unternehmen halten will oder kann. Falsche Offerten kippen nämlich leicht in unattraktive Geschäfte um.

\section{Fazit}

Raus aus der engen Schublade bei Kunden! Diese Herausforderung ist verbreitet, aber bisher zu wenig thematisiert. Unternehmen können für Kunden meist weit mehr leisten, als diese beanspruchen.

Fakt ist: Es gibt keine einfachen Hebel, um aus der engen Schublade bei Kunden rauszukommen. Auch dieser Beitrag zeigt keine klare Lösung, die Probleme sind zu vielschichtig. Es lohnt sich aber für Unternehmen, diese Problematik spezifisch anzugehen. Zuerst braucht es dazu Fakten und nicht nur Meinungen und Hoffnungen.

\section{a Zusätzlicher Verlagsservice für Abonnenten von „Springer für Professionals | Vertrieb“}

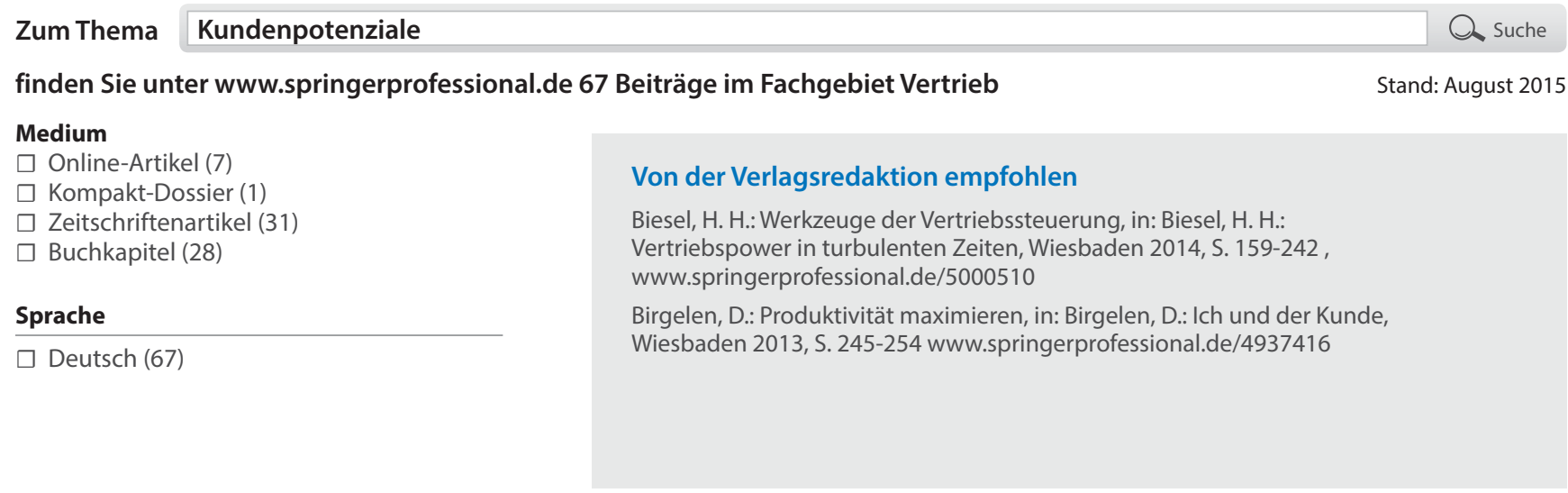

